\title{
Phenotypic and genetic spectrum of isolated macrodactyly: somatic mosaicism of PIK3CA and $A K T 1$ oncogenic variants
}

\author{
Wen Tian ${ }^{1 \dagger}$, Yingzhao Huang ${ }^{2,3,4 \dagger}$, Liying Sun', Yang Guo', Sen Zhao ${ }^{2,3,4}$, Mao Lin 2,3,4, Xiying Dong 2,3,4, \\ Wenyao Zhong', Yuehan Yin' ', Zefu Chen 2,3,4, Nan Zhang ${ }^{1}$, Yuanqiang Zhang 2,3,4, Lianlei Wang ${ }^{2,3,4}$, \\ Jiachen Lin ${ }^{2,3,4}$, Zihui Yann 2,3,4 Xinzhuang Yang ${ }^{5}$, Junhui Zhao ${ }^{1}$, Guixing Qiu 2,3,4, Jianguo Zhang 2,3,4, \\ Zhihong $\mathrm{Wu}^{3,4,5^{*}}$ and Nan W $\mathrm{u}^{2,3,4^{*}}$ (D) on behalf of (Deciphering Disorders Involving Scoliosis, COmorbidities) \\ study group
}

\begin{abstract}
Background: Isolated macrodactyly is a severe congenital hand anomaly with functional and physiological impact. Known causative genes include PIK3CA, AKT1 and PTEN. The aim of this study is to gain insights into the genetics basis of isolated macrodactyly.

Results: We enrolled 24 patients with isolated macrodactyly. Four of them were diagnosed with Proteus syndrome based on skin presentations characteristic to this disease.Targeted next-generation sequencing was performed using patients' blood and affected tissues. Overall, 20 patients carry mosaic PIK3CA pathogenic variants, i.e. p.His 1047 Arg $(N=7)$, p.Glu542Lys $(N=6)$, p.Glu545Lys $(N=2)$, p.His1047Leu $(N=2)$, p.Glu453Lys $(N=1)$, p.GIn546Lys $(N=1)$ and p. His 1047Tyr $(\mathrm{N}=1)$. Four patients who met the diagnostic criteria of Proteus syndrome carry mosaic AKT1 p.Glu17Lys variant. Variant allele frequencies of these mosaic variants obtained through next-generation sequencing range from 10 to $33 \%$. In genotype-phenotype correlation analysis of patients with PIK3CA variant, we found that patients with the macrodactyly of the lower limbs tend to carry PIK3CA variants located in the helical domain $(P=0.005)$.

Conclusions: Mosaic PIK3CA and AKT1 variants can be found in all of our samples with isolated macrodactyly. Insights into phenotypic and genetic spectrum of isolated macrodactyly may be helpful in perusing a more precise and effective management of isolated macrodactyly.
\end{abstract}

Keywords: Macrodactyly, Phosphatidylinositol 3-kinase catalytic subunit alpha (PIK3CA), AKT1 serine/threonine kinase 1 (AKT1), Somatic mosaicism, Proteus syndrome

\footnotetext{
*Correspondence: orthoscience@126.com; dr.wunan@pumch.cn

${ }^{\dagger}$ Wen Tian and Yingzhao Huang contributed equally to this work

2 Department of Orthopedic Surgery, Peking Union Medical College Hospital, Peking Union Medical College, Chinese Academy of Medical Sciences, No. 1 Shuaifuyuan, Beijing 100730, China

${ }^{5}$ Department of Central Laboratory, Peking Union Medical College Hospital, Peking Union Medical College, Chinese Academy of Medical Sciences, No. 1 Shuaifuyuan, Beijing 100730, China

Full list of author information is available at the end of the article
}

\begin{abstract}
Introduction
Macrodactyly is a rare congenital anomaly characterized by the overgrowth of digits on one or multiple limbs, which can present as either isolated or syndromic (in conjunction with other congenital defects) macrodactyly. It occurs in approximately $1 / 50,000-1 / 100,000$ live births, and varies according to regional and ethnical demographics $[1,2]$. The associated dysmorphic appearance and cultural stigma have the potential to lead to psychological and social problems that can persist through
\end{abstract}


to adulthood. Due to the highly variable phenotypic manifestation and low frequency of occurrence, there is no standardized protocol for the treatment of macrodactyly, presenting a unique challenge for surgeons faced with correcting abnormalities [3]. Currently, surgical procedures such as soft tissue debulking, physeal arrest and amputation are used to produce acceptable functional and cosmetic outcomes, but novel therapeutic strategies are still needed [5].

Somatic mosaicism of phosphatidylinositol 3-kinase catalytic subunit alpha (PIK3CA) mutations has been identified as the cause of multiple overgrowth disorders, including isolated macrodactyly, congenital lipomatous overgrowth, vascular malformations, epidermal nevi and skeletal/scoliosis/spinal abnormalities (CLOVES syndrome) [4], Kippel-Trenaunay syndrome (KTS) [5], megalencephaly-capillary malformation (MCAP) [6], dysplastic megalencephaly (DMEG) [7], and capillary malformation of the lower lip, lymphatic malformation of the face and neck, and asymmetry and partial/generalized overgrowth (CLAPO syndrome) [8]. Despite their clinical differences, tissue enlargement represents a distinct feature common to all of these disorders. PIK3CA is one of the most frequently mutated genes in human tumors [9], encoding the alpha catalytic subunit of phosphatidylinositol-4,5-bisphosphate 3-kinase, a member of the phosphatidylinositol 3-kinase (PI3K) enzyme family [9]. Signaling through PI3K-AKT-mTOR mediates cell proliferation, survival and metabolism through enhancement of lipid kinase activity, and constitutive activation of this pathway through mutations in PI $3 K 3 C A$ is well established in tumorigenesis [10].

While the correlation between PIK3CA and AKT1 mutations in macrodactyly is well characterized [11], the mutational architecture of somatic mosaicism and its effect on phenotypic presentation in isolated macrodactyly is still not well understood, particularly due to the challenges of identifying of low-level mosaicism in affected tissue. Currently, research employing next generation sequencing (NGS)-based methods to explore the genetic basis of isolated macrodactyly hold much promise. In this study, we utilize an NGS-based strategy to identifiy mosaic PIK3CA and $A K T 1$ variants in a cohort of 24 isolated macrodactyly patients. Furthermore, we assess the genotype-phenotype correlation caused by distinct PIK3CA variants in isolated macrodactyly.

\section{Results}

\section{Clinical characteristics of the subjects}

Clinical and molecular characteristics of these 24 subjects are described in Table 1. Representative photographs of nine isolated macrodactyly patients are shown in Fig. 1. 22 of 24 patients displayed asymmetric and disproportionate overgrowth in their hands or feet at birth, while the remaining 2 patients had later onset symptoms between six and twelve months after birth. With the exception of one 34 years old, the majority of patients were under 15 years old, with a median age of 5 . A slight male predominance was observed (16 males vs. 8 females, binomial $P=0.152$ ) in our cohort. 14 patients presented exclusive involvement of the lower extremities, while nine had macrodactyly exclusively in the upper extremities (binomial $P=0.405$ ). Only one patient had overgrowth in both the upper and the lower extremities. Twenty of the 24 patients had unilateral involvement, with 12 patients presenting on the right side and eight patients presenting on the left side of the body (binomial $P=0.503)$. The number of affected digits ranged from one to seven, with an average of 2.7 digits affected. The second digit was the most frequently affected digit $(\mathrm{N}=22)$, followed by the third $(\mathrm{N}=17)$. Over half of our patients $(14 / 24)$ had two affected digits, and the combination of second- and third-digit overgrowth $(\mathrm{N}=10)$ was more frequently observed than the combination of first- and second-digit enlargement $(\mathrm{N}=4$; binomial $P=0.180$ ). In addition, no other combinations were observed in patients with two affected digits. 5 of the 24 patients had syndactyly, a condition where two or more digits are fused together, all of which presented as syndactyly of $2-3$ toes.

Patients 1-4 had variable presentations of cerebriform connective tissue nevi adjacent to overgrown digits. Therefore, diagnoses of Proteus syndrome were established in these patients.

\section{Genetic characteristics}

Through genetic testing and analysis, we achieved molecular diagnoses of all 24 patients. In the 4 patients who met the diagnostic criteria of Proteus syndrome, we identified an AKT1 c.49G > A (p.Glu17Lys) variant, which is the only variant known to cause Proteus syndrome [12]. Variant allele frequencies (VAFs) of this $A K T 1$ mutation in affected tissues ranged from 10 to $22 \%$, with an average variant frequency of $16 \%$. No variant read was identified in blood DNA (Table 1).

In the 20 patients with isolated macrodactyly who did not meet the diagnostic criteria for Proteus syndrome, we identified and confirmed pathogenic variants in PIK3CA (Table 1). The most commonly observed variant was PIK3CA p.His1047Arg $(\mathrm{N}=7)$, followed by p.Glu542Lys $(\mathrm{N}=6)$, p.Glu545Lys $(\mathrm{N}=2)$, p.His1047Leu $(\mathrm{N}=2)$, p.Glu453Lys $(\mathrm{N}=1)$, p.Gln546Lys $(\mathrm{N}=1)$ and p.His1047Tyr $(\mathrm{N}=1)$ (Table 1). VAFs in affected tissues ranged from 10 to $33 \%$ with an average of $21 \%$ (Table 1). None of these variants were identified in peripheral blood samples. 
Table 1 Summary of the clinical and molecular findings of studied subjects

\begin{tabular}{|c|c|c|c|c|c|c|c|c|}
\hline Patient nos. & Gender & Age & Syndactyly & Skin findings & Affected digits & Gene & Variant & VAF (\%) \\
\hline 1 & M & 11 & N & CCTN & R-Hand: 2,3 & AKT1 & c.49G > A (p.Glu17Lys) & 22.03 \\
\hline 2 & M & 11 & $\mathrm{~N}$ & CCTN & L-Foot: $1,2,3,4,5$ & AKT1 & c.49G > A (p.Glu17Lys) & 11.16 \\
\hline 3 & M & 2 & N & CCTN & R-Hand: 2,3 & AKT1 & c.49G > A (p.Glu17Lys) & 9.93 \\
\hline 4 & $\mathrm{~F}$ & 10 & $\mathrm{~N}$ & CCTN & L-Hand: $3,4,5$ & AKT1 & c.49G > A (p.Glu17Lys) & 20.57 \\
\hline 5 & F & 6 & N & N & L-Foot: 2,3; R-Foot:1.2; R-Hand:2,3 & PIK3CA & c.1357G > A (p.Glu453Lys) & 11.10 \\
\hline 6 & M & 3 & $\mathrm{~N}$ & N & R-Foot: 2 & PIK3CA & c.1624G > A (p.Glu542Lys) & 24.48 \\
\hline 7 & M & 4 & Y & N & L-Foot: 2,3 & PIK3CA & c.1624G > A (p.Glu542Lys) & 17.15 \\
\hline 8 & M & 5 & Y & N & L-Foot: 1,2,3,4 & PIK3CA & c.1624G > A (p.Glu542Lys) & 20.95 \\
\hline 9 & M & 4 & $\mathrm{~N}$ & N & L-Foot: 2,3 & PIK3CA & c.1624G > A (p.Glu542Lys) & 17.10 \\
\hline 10 & $\mathrm{~F}$ & 4 & $\mathrm{~N}$ & N & R-Foot: 2,3 & PIK3CA & c.1624G > A (p.Glu542Lys) & 27.58 \\
\hline 11 & M & 3 & $\mathrm{~N}$ & N & R-Foot: 1,2 & PIK3CA & c.1624G > A (p.Glu542Lys) & 17.79 \\
\hline 12 & M & 2 & $\mathrm{~N}$ & N & L-Foot: 1,2,3 & PIK3CA & c.1633G > A (p.Glu545Lys) & 19.11 \\
\hline 13 & M & 2 & Y & N & R-Foot: 2,3 & PIK3CA & c.1633G > A (p.Glu545Lys) & 27.31 \\
\hline 14 & M & 11 & $N$ & N & L-Foot: 1,2,3; R-Foot:1,2,3,4 & PIK3CA & c.1636C > A (p.Gln546Lys) & 24.50 \\
\hline 15 & $\mathrm{~F}$ & 13 & $\mathrm{~N}$ & N & B-Feet: 2 & PIK3CA & c.3139C > T (p.His1047Tyr) & 18.94 \\
\hline 16 & M & 1 & N & N & R-Hand: 2,3 & PIK3CA & c.3140A > G (p.His1047Arg) & 25.63 \\
\hline 17 & M & 2 & Y & N & R-Foot: 1,2 & PIK3CA & c.3140A > G (p.His1047Arg) & 23.29 \\
\hline 18 & $\mathrm{~F}$ & 5 & Y & $\mathrm{N}$ & L-Foot: 2,3 & PIK3CA & c.3140A > G (p.His1047Arg) & 21.45 \\
\hline 19 & M & 2 & N & N & R-Hand: 1,2 & PIK3CA & c.3140A > G (p.His1047Arg & 25.57 \\
\hline 20 & $\mathrm{~F}$ & 10 & N & $\mathrm{N}$ & R-Hand: $1,2,3,4,5$ & PIK3CA & c.3140A > G (p.His1047Arg) & 10.36 \\
\hline 21 & M & 2 & N & $\mathrm{N}$ & L-Foot: 2,3 & PIK3CA & c.3140A > G (p.His1047Arg) & 20.03 \\
\hline 22 & $\mathrm{~F}$ & 6 & $\mathrm{~N}$ & $\mathrm{~N}$ & L-Hand: 5; R-Hand:1,2,3 & PIK3CA & c.3140A > G (p.His1047Arg) & 15.82 \\
\hline 23 & $\mathrm{~F}$ & 3 & $\mathrm{~N}$ & $\mathrm{~N}$ & R-Hand: 5 & PIK3CA & c.3140A > T (p.His1047Leu) & 33.38 \\
\hline 24 & M & 34 & $\mathrm{~N}$ & $\mathrm{~N}$ & R-Hand: 1,2 & PIK3CA & c.3140A >T (p.His1047Leu) & 18.99 \\
\hline
\end{tabular}

$M$ male, $F$ female, $Y$ yes or present, $N$ not present, $L$ left, $R$ right, $B$ bilateral, VAF variant allele frequency, $N A$ not available, $C C T N$ cerebriform connective tissue nevi

All seven variants have been previously reported to cause developmental disorders [10, 11, 13, 14], and either predicted or validated to have gain-of-function mechanisms. In vivo studies have demonstrated that mutations in PIK3CA are sufficient to induce oncogenic transformation in chicken embryo fibroblasts through enhancement lipid kinase activity and activation of mTOR and AKT1 signaling [15]. All seven variants were absent from the Deciphering Disorders Involving Scoliosis and COmorbidities (DISCO, https://discostudy .org/) study composed of 4000 exome sequencing data of the Chinese population [16-18]. PIK3CA p.Glu453Lys, p.Glu542Lys, p.Gln546Lys and p.His1047Tyr were absent from the Genome Aggregation Database (gnomAD, https ://gnomad.broadinstitute.org). PIK3CA p.Glu545Lys, p.His1047Leu, p.His1047Arg and $A K T 1$ p.Glu17Lys were present at extremely low frequencies in gnomAD, with an allele frequency of 4e-6. Despite previously reported observations of PIK3CA p.Gln546Lys, p.His1047Tyr and p.Glu453Lys mutations in other PIK3CA-related overgrowth syndromes (PROS) [11, 13, 19-21], our study represents the first time they are identified in isolated macrodactyly.
The PI3K protein has five functional domains, including PI3K-ABD, PI3K-RBD, C2 PI3K-type, PIK helical and PI3K/PI4K kinase domain. The p.Glu453Lys mutation is located in the $\mathrm{C} 2$ domain. Meanwhile, the p.Glu542Lys, p.Glu545Lys and p.Gln546Lys mutations occurr in adjacent amino acids of the helical domain. Lastly, the p.His1047Arg, p.His1047Tyr and p.His1047Leu mutations are located at the kinase domain of PIK3CA (Fig. 2). The majority of established functional variants of the PIK3CA cluster were found in the kinase and helical domains [22], which is consistent with our findings. In the 20 patients carrying pathogenic PIK3CA variants, 9 had variants of the helical domain and 10 had patients affecting the kinase domain, and only one patient had a variant affecting the $\mathrm{C} 2$ domain.

\section{Genotype-phenotype correlation}

We then analyzed the potential correlation between subjects' phenotypes (i.e. macrodactyly of the upper or the lower limb, the number of affected digits, with or without syndactyly) and the domain location of variant mutations in patients carrying the PIK3CA variant (Table 2). 


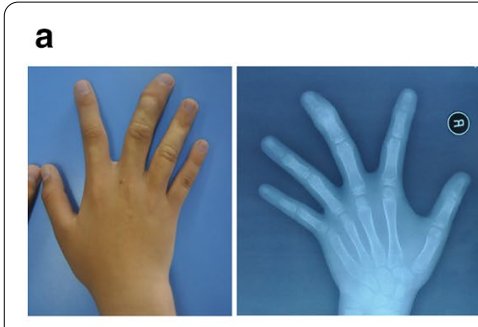

d

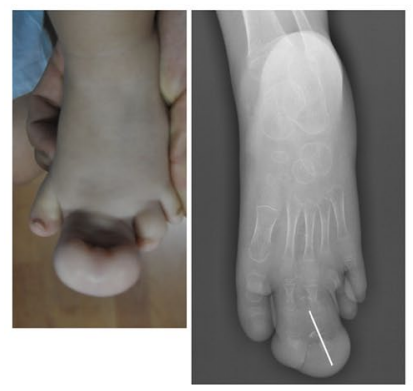

g

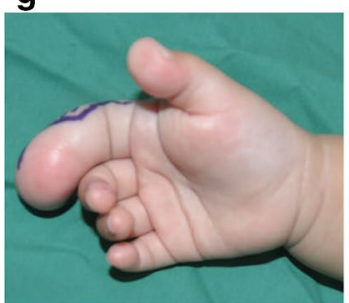

b

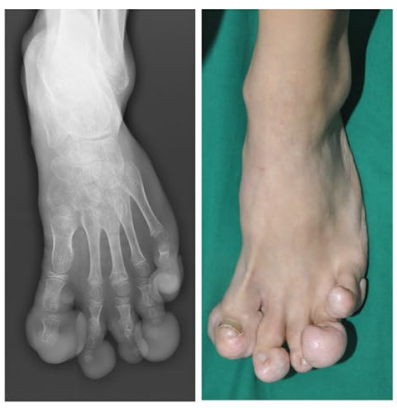

e

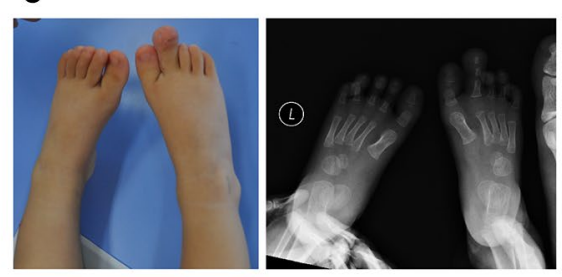

h

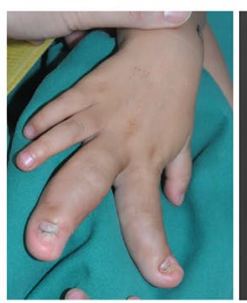

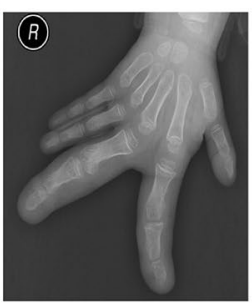

C

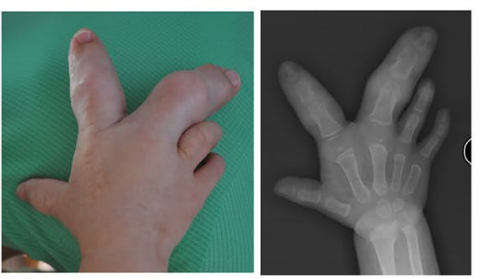

f
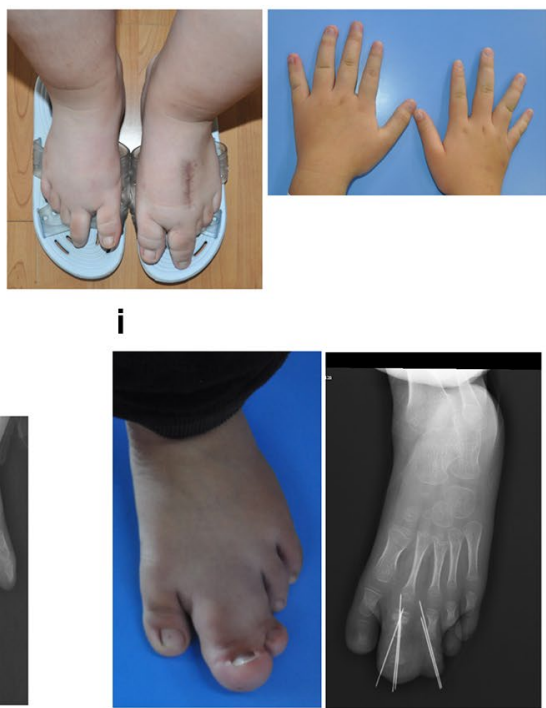

Fig. 1 Representative clinical photographs of nine macrodactyly patients. a Patient No. 1. b Patient No. 2. c Patient No. 3. d Patient No. 18. e Patient No. 6. f Patient No. 15. g Patient No. 19. h Patient No. 16. i Patient No. 21

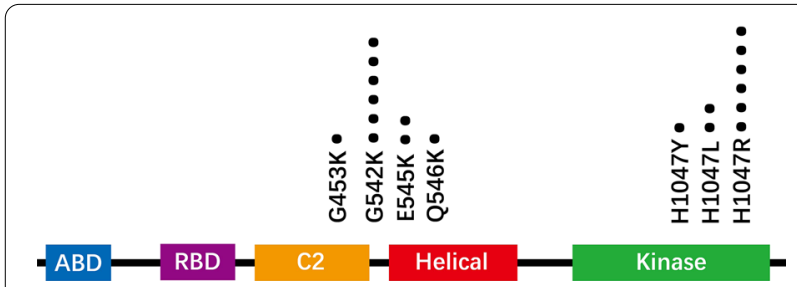

Fig. 2 Distribution of PIK3CA variants identified in this study by functional domains. ABD: PI3K-ABD domain. RBD: PI3K-RBD domain. C2: C2 PI3K-type domain. Helical: PIK helical domain. Kinase: PI3K/PI4K kinase domain. A dot represents a PIK3CA variant identified in one patient

All $9(100 \%)$ patients carrying variants in the helical domain of PI3K presented with macrodactyly in the lower limbs. In contrast, only 4 of 13 (36\%) patients carrying variants outside of the helical domain (in the C2 domain or kinase domain) had a lower limb affected. These data demonstrates that helical domain variants are enriched in patients with lower limb overgrowth relative to all other variants $(P=0.005$; determined by Fisher's
Table 2 Genotype-phenotype correlation

\begin{tabular}{lll}
\hline & In helical domain & $\begin{array}{l}\text { Not } \\
\text { in helical } \\
\text { domain }\end{array}$ \\
\hline $\begin{array}{l}\text { Affected limb } \\
\text { Upper limb or both } \\
\begin{array}{l}\text { Lower limb } \\
\text { P-value }\end{array}\end{array}$ & 0 & 7 \\
$\begin{array}{l}\text { Affected digits } \\
<3\end{array}$ & 9 & 4 \\
$\geq 3$ & 0.005 & 8 \\
$P$-value & & 3 \\
\hline
\end{tabular}

exact test). We also compared the VAFs between the two phenotypically different groups, but no significant correlation was observed.

13 patients had one or two affected digits, and five (38\%) of these patients carried PIK3CA mutations located in the helical domain. 7 patients had more than two affected digits, 4 (57\%) of whom had a PIK3CA 
mutation in the helical domain. While it may appear at first glance that patients with less than three affected digits were more likely to have a variant not located in the helical domain, and vice versa, this observation did not prove to be statistically significant $(P=0.642$; determined by Fisher's exact test).

\section{Discussion}

In this study, 24 patients with macrodactyly underwent a targeted NGS-based sequencing and were identified to harbor mosaicism of either a pathogenic PIK3CA or $A K T 1$ variant. No pathogenic or likely-pathogenic variant was detected in other genes currently known to be associated with macrodactyly, implicating somatic mosaicism of PIK3CA or AKT1 mutations as a predominant cause of isolated macrodactyly. This inference is supported by a separate recent study in which 9 out of 12 subjects with non-syndromic macrodactyly were positive for somatic mosaicism in PIK3CA [19].

Previous studies on the genetic causes of macrodactyly have largely applied Sanger sequencing toward mosaic variant detection [12, 23], which is not sensitive enough to detect low-level mosaicism [23]. Indeed, the mosaicism in our study patients no. 2, 3, 5, 8 and 20 would have been unlikely to be identified through this method (Additional file 1).In this report, we demonstrate that an NGS-based method can detect mosaicism even as low as $10 \%$. Due to the limitations of Sanger sequencing in identifying mosaic variants and the wide spectrum of pathogenic variants (eight distinct variants identified in this study) [24], we employed NGS-based deep sequencing as our first-line in the molecular diagnosis of isolated macrodactyly.

PIK3CA mutations have been associated with a wide group of overgrowth disorders, with overlapping clinical manifestations. Macrodactyly has been reported in patients with CLAPO syndrome [8], CLOVES syndrome [4], KTS [25], as well as some forms of isolated lymphatic malformation or muscular hypertrophy [26]. CLAPO syndrome is characterized by capillary malformation of the lower lip, lymphatic malformation of the face and neck, and asymmetric overgrowth. In contrast, CLOVES syndrome presents with congenital lipomatous overgrowth, vascular malformations, epidermal nevi, and skeletal/scoliosis and spinal abnormalities. KTS is characterized by a triad of capillary malformation, venous varicosities and limb hypertrophy [27]. To differentially diagnose these separate PROS, clinicians must carefully evaluate patient's phenotypes, as proper diagnosis has important implications in clinical management and follow-up.

Consistent with our report, the most commonly observed PIK3CA somatic variants in PIK3CA-related overgrowth syndromes (PROS) are p.His1047Arg, p.His1047Leu, p.Glu545Lys and p.Glu542Lys [13]. There are remarkable discrepancies between measured mosaic levels of samples collected from the same patient depending on whether Sanger sequencing or NGS is used [13, 26]. Seven variants of PIK3CA and one variant of $A K T 1$ that were identified in this study were previously described $[13,28]$. However, we observed a significant heterogeneity in phenotypic presentations even for the same variant, which might be a consequence of the different times during development at which mutations were acquired [29]. The exact sites of variant-induced affliction in the human body might also play a role. It has been observed that PROS not involving the brain are usually caused by PIK3CA cancer hot-spot variants, e.g. p.Glu542Lys, p.His1047Arg and p.Glu453Lys [11, 13], while PROS involving the brain are usually caused by relatively rare variants. This phenomenon is also described in our report. Furthermore, we showed that macrodactyly in the upper limbs is caused primarily by variants outside the helical domain, whereas PROS involving the lower limbs are often a result of variants in the helical domain, and this association shows a robust statistical significance $(P=0.005)$. To evaluate the reproducibility of this result, we searched previous publications and found 13 patients from two studies with sufficient information to analyze genotype-phenotype correlation $[14,19]$. While this sample size was too small to return a significant association $(P=0.497)$, the trend remains that all patients with mutations in the helical domain have only lower limbs affected. Indeed, when our data is combined with this previous data, the correlation is statistically significant $(P=0.002)$. Nevertheless, replication of this result in an independent cohort is still needed.

We found that VAFs of PIK3CA and AKT1 in affected tissues were around $20 \%$, and no variant was identified in blood samples, further supporting previous findings which suggests that PIK3CA mutations are generally undetectable in the blood of patients with Proteus syndrome or with PROS, excluding MCAP [12, 23, 30].

In conclusion, our findings demonstrate that isolated macrodactyly is predominantly caused by mosaic variants in PIK3CA or AKT1. We also show that patients with macrodactyly deformities of the upper limbs tend to carry PIK3CA variants with mutations outside the helical domain, while patients with deformities in the lower limbs have PIK3CA variants with mutations in the helical domain. With the advent of targeted therapy against the PI3K-AKT1-mTOR pathway, a deeper understanding of the pathogenesis of isolated macrodactyly holds great potential to benefit its effective management. 


\section{Conclusions}

This study reports the largest series of patients so far with PIK3CA /AKT1-associated macrodactyly, which includes 24 patients with isolated macrodactyly, all of whom carry mosaic PIK $3 C A$ or $A K T 1$ variants. Our findings expand the understanding of the mutational architecture and identifies novel genotype-phenotype correlations, providing insight into the genetic etiology of mosaic overgrowth syndromes and lends hope to improving precise management of these sets of syndromes.

\section{Methods}

\section{Patient selection}

Our study recruited 24 subjects clinically presenting with isolated macrodactyly, who were admitted to Jishuitan Hospital in 2018. Our criteria was limited to patients whose overgrowth did not exceed the limbs. Four subjects met the diagnostic criteria for Proteus syndrome and presented with skin findings and overgrowth limited to the hand or foot. The limb anomalies of these patients were evaluated through physical examinations and $\mathrm{x}$-ray by experienced hand surgeons (WT, LS and YG).

\section{Tissue sampling and preparation}

Abnormal adipose, skin and nerve tissues were collected during surgery. Genomic DNA was extracted from blood and collected tissues using the Dneasy Blood and Tissue Kit (QIAGEN, Germany) according to the manufacturer's protocol.

\section{Genetic test and variant interpretation}

A deep-targeted NGS protocol was performed on DNA extracted from blood and from surgically-removed abnormal tissues of all 24 subjects. Illumina paired-end libraries were prepared from DNA samples and were subjected to a customized NGS panel to detect somatic mutations on the mTOR-pathway-related genes, including $A K T 1, A K T 2, A K T 3, P I K 3 C A$ and MTOR, on the Illumina HiSeq X Ten platform (Illumina, US). The mean coverage for these mTOR-pathway-related genes was 1000 reads with more than $98 \%$ regions exceeding 100 reads. In-house developed Peking Union Medical College Hospital Pipeline (PUMP) and variant interpretation were performed following previously described methods [16-18].

All variants presumed to be pathogenic were subjected to Sanger sequencing. Variant-encoding amplicons were amplified by PCR from genomic DNA obtained from subjects, purified using an Axygen AP-GX-50 kit (lot no.
05915KE1) and sequenced by Sanger sequencing on an ABI3730XL instrument.

\section{Genotype-phenotype correlation analysis}

Patients carrying variants in PIK3CA gene were selected to analyze potential genotype-phenotype correlation. We divided the patients into two groups based on the respective domain of an individual's variant. We then compared the location of affected sites (at the upper or lower limbs) and the number of affected digits between the two groups.

SPSS Statistics V15.0 software was used for statistical analyses, and a p-value lower than 0.05 was considered statistically significant. Genotype-phenotype correlation was assessed using Fisher's exact test and Student's t test.

\section{Supplementary information}

Supplementary information accompanies this paper at https://doi. org/10.1186/s13023-020-01572-9.

Additional file 1. Results of Sanger sequencing of the variants in AKT1 and PIK3CA.

\section{Abbreviations}

PIK3CA: Phosphatidylinositol 3-kinase catalytic subunit alpha; AKT1: AKT serine/threonine kinase 1; PI3K: Phosphatidylinositol 3-kinase; NGS: Next generation sequencing; VAFs: Variant allele frequencies; PROS: PIK3CA-related overgrowth syndrome; MCAP: Megalencephaly-capillary malformation.

\section{Acknowledgements}

We appreciate all of the patients, their families and clinical staffs.

\section{Authors' contributions}

NW, ZW, GQ, JZ and JZ conceived and designed the study. WT, LS, GY, ML, $Y Y, N Z, W Z$ and $Y H$ enrolled the cohort. JL, ZC, LW, YZ and YH conducted the experiments. WT, YH, LS, SZ and WN analyzed the data. ZY, XD and ZC conducted the bioinformatic analyses. XY, JZ, GQ and ZW assisted with study organization and manuscript revision. $Z \mathrm{~W}$ and $J Z$ assisted with data interpretation. WT, YH, LS, YG, SZ, XD, XG and NW wrote the manuscript. All authors read and approved the final manuscript.

\section{Funding}

This research was funded in part by the The National Key Research and Development Program of China (2016YFC0901500 and 2018YFC0910506), Center for Rare Diseases Research, Chinese Academy of Medical Sciences, Beijing, China (2016ZX310174-4), Beijing JST Research Funding (ZR-201907 and 2019YJ03), Beijing Jishuitan Hospital Nova Program (XKXX201818), National Natural Science Foundation of China (81822030, 81772299, 81930068, 81772301, 81972132, 81672123 and 81972037), Beijing Natural Science Foundation (7172175 and 7191007) and the Tsinghua University-Peking Union Medical College Hospital Initiative Scientific Research Program, CAMS Initiative Fund for Medical Sciences (2016-I2M-3-003, 2016-I2M-2-006 and 2017-I2M-2-001).

\section{Availability of data and materials}

The datasets used and analyzed during the current study are available from the corresponding author on reasonable request.

Ethics approval and consent to participate

Informed consent was acquired during this study. This study is approved by the ethnic committee of Peking Union Medical College Hospital.

Consent for publication

The consent for publication was acquired from patients or patients' parents. 


\section{Competing interests}

The authors declare that they have no competing interests.

\section{Author details}

${ }^{1}$ Department of Hand Surgery, Beijing Jishuitan Hospital, Beijing 100035, China. ${ }^{2}$ Department of Orthopedic Surgery, Peking Union Medical College Hospital, Peking Union Medical College, Chinese Academy of Medical Sciences, No. 1 Shuaifuyuan, Beijing 100730, China. ${ }^{3}$ Beijing Key Laboratory for Genetic Research of Skeletal Deformity, Beijing 100730, China. ${ }^{4}$ Medical Research Center of Orthopedics, Chinese Academy of Medical Sciences, Beijing 100730, China. ${ }^{5}$ Department of Central Laboratory, Peking Union Medical College Hospital, Peking Union Medical College, Chinese Academy of Medical Sciences, No. 1 Shuaifuyuan, Beijing 100730, China.

Received: 24 December 2019 Accepted: 5 October 2020 Published online: 14 October 2020

\section{References}

1. Giele H, Giele C, Bower C, Allison M. The incidence and epidemiology of congenital upper limb anomalies: a total population study. J Hand Surg Am. 2001;26:628-34.

2. Koskimies E, Lindfors N, Gissler M, Peltonen J, Nietosvaara Y. Congenital upper limb deficiencies and associated malformations in finland: a population-based study. J Hand Surg. 2011;36:1058-65.

3. Gluck JS, Ezaki M. Surgical treatment of macrodactyly. J Hand Surg. 2015:40:1461-8.

4. Martinez-Lopez A, Blasco-Morente G, Perez-Lopez I, Herrera-Garcia JD, Luque-Valenzuela M, Sanchez-Cano D, et al. CLOVES syndrome: review of a PIK3CA-related overgrowth spectrum (PROS). Clin Genet. 2017;91:14-21.

5. Vahidnezhad $\mathrm{H}$, Youssefian $\mathrm{L}$, Uitto J. Klippel-Trenaunay syndrome belongs to the PIK3CA-related overgrowth spectrum (PROS). Experim Dermatol. 2016:25:17-9.

6. Mirzaa GM, Conway RL, Gripp KW, Lerman-Sagie T, Siegel DH, deVries LS, et al. Megalencephaly-capillary malformation (MCAP) and megalencephaly-polydactyly-polymicrogyria-hydrocephalus (MPPH) syndromes: two closely related disorders of brain overgrowth and abnormal brain and body morphogenesis. Am J Med Genet Part A. 2012;158:269-91.

7. Jansen LA, Mirzaa GM, Ishak GE, O'Roak BJ, Hiatt JB, Roden WH, et al. PI3K AKT pathway mutations cause a spectrum of brain malformations from megalencephaly to focal cortical dysplasia. Brain. 2015;138:1613-28.

8. Rodriguez-Laguna L, Ibañez K, Gordo G, Garcia-Minaur S, Santos-Simarro F, Agra N, et al. CLAPO syndrome: identification of somatic activating PIK3CA mutations and delineation of the natural history and phenotype. Genet Med. 2018;20:882-9.

9. Samuels Y, Wang Z, Bardelli A, Silliman N, Ptak J, Szabo S, et al. High frequency of mutations of the PIK3CA gene in human cancers. Science. 2004;304:554.

10. Samuels $Y$, Ericson K. Oncogenic PI3K and its role in cancer. Curr Opin Oncol. 2006;18:77-82.

11. Kuentz P, St-Onge J, Duffourd Y, Courcet J-B, Carmignac V, Jouan T, et al. Molecular diagnosis of PIK3CA-related overgrowth spectrum (PROS) in 162 patients and recommendations for genetic testing. Genet Med. 2017;19:989-97.

12. Lindhurst MJ, Sapp JC, Teer JK, Johnston JJ, Finn EM, Peters K, et al. A mosaic activating mutation in AKT1 associated with the Proteus syndrome. N Engl J Med. 2011;365:611-9.

13. Mirzaa G, Timms AE, Conti V, Boyle EA, Girisha KM, Martin B, et al. PIK3CAassociated developmental disorders exhibit distinct classes of mutations with variable expression and tissue distribution. JCI Insight (Internet). 2016 (cited 2018 Oct 3);1. https://insight.jci.org/articles/view/87623.

14. Keppler-Noreuil KM, Sapp JC, Lindhurst MJ, Parker VER, Blumhorst C, Darling T, et al. Clinical delineation and natural history of the PIK3CA-related overgrowth spectrum. Am J Med Genet. 2014;164:1713-33.

15. Gymnopoulos M, Elsliger M-A, Vogt PK. Rare cancer-specific mutations in PIK3CA show gain of function. PNAS. 2007:104:5569-74.

16. Wang K, Zhao S, Liu B, Zhang Q, Li Y, Liu J, et al. Perturbations of BMP/ TGF- $\beta$ and VEGFNEGFR signalling pathways in non-syndromic sporadic brain arteriovenous malformations (BAVM). J Med Genet. 2018;55:675-84.

17. Wang K, Zhao S, Zhang Q, Yuan J, Liu J, Ding X, et al. Whole-exome sequencing reveals known and novel variants in a cohort of intracranial vertebral-basilar artery dissection (IVAD). J Hum Genet. 2018;63:1119-28.

18. Liu J, Zhou Y, Liu S, Song X, Yang X-Z, Fan Y, et al. The coexistence of copy number variations (CNVs) and single nucleotide polymorphisms (SNPS) at a locus can result in distorted calculations of the significance in associating SNPs to disease. Hum Genet. 2018;137:553-67.

19. Wu J, Tian W, Tian G, Sumner K, Hutchinson DT, Ji Y. An investigation of PIK3CA mutations in isolated macrodactyly. J Hand Surg (Eur Vol). 2018:43:756-60.

20. Tripolszki K, Knox R, Parker V, Semple R, Farkas K, Sulák A, et al. Somatic mosaicism of the PIK3CA gene identified in a Hungarian girl with macrodactyly and syndactyly. Eur J Med Genet. 2016;59:223-6.

21. Rios JJ, Paria N, Burns DK, Israel BA, Cornelia R, Wise CA, et al. Somatic gain-of-function mutations in PIK3CA in patients with macrodactyly. Hum Mol Genet. 2013;22:444-51.

22. Burke JE, Perisic O, Masson GR, Vadas O, Williams RL. Oncogenic mutations mimic and enhance dynamic events in the natural activation of phosphoinositide 3-kinase p110 (PIK3CA). Proc Natl Acad Sci. 2012;109:15259-64

23. Rivière J-B, Mirzaa GM, O'Roak BJ, Beddaoui M, Alcantara D, Conway RL, et al. De novo germline and postzygotic mutations in AKT3, PIK3R2 and PIK3CA cause a spectrum of related megalencephaly syndromes. Nat Genet. 2012;44:934-40.

24. Spinner NB, Conlin LK. Mosaicism and clinical genetics. Am J Med Genet C Semin Med Genet. 2014;166C:397-405.

25. Sung HM, Chung HY, Lee SJ, Lee JM, Huh S, Lee JW, et al. Clinical experience of the Klippel-Trenaunay syndrome Archives of plastic surgery. Korean Soc Plastic Reconstr Surg. 2015;42:552.

26. Luks VL, Kamitaki N, Vivero MP, Uller W, Rab R, Bovée JVMG, et al. Lymphatic and other vascular malformative/overgrowth disorders are caused by somatic mutations in PIK3CA. J Pediatr. 2015;166:1048-1054.e1-5.

27. Mullins JF, Naylor D, Redetski J. The Klippel-Trenaunay-Weber syndrome: naevus vasculosus osteohypertrophicus. Arch Dermatol. 1962;86:202-6.

28. Blesinger $H$, Kaulfuß S, Aung T, Schwoch S, Prantl L, Rößler J, et al. PIK3CA mutations are specifically localized to lymphatic endothelial cells of lymphatic malformations. PLoS ONE. 2018;13:e0200343.

29. Frisk S, Taylan F, Blaszczyk I, Nennesmo I, Annerén G, Herm B, et al. Early activating somatic PIK3CA mutations promote ectopic muscle development and upper limb overgrowth. Clin Genet. 2019;96:118-25.

30. Keppler-Noreuil KM, Rios JJ, Parker VER, Semple RK, Lindhurst MJ, Sapp JC, et al. PIK3CA-related overgrowth spectrum (PROS): diagnostic and testing eligibility criteria, differential diagnosis, and evaluation. Am J Med Genet A. 2015;167A:287-95.

\section{Publisher's Note}

Springer Nature remains neutral with regard to jurisdictional claims in published maps and institutional affiliations. 